\title{
New scheme to overcome local optima in the Voxel-Based Topology Optimization of Sheet Metal Structures
}

\author{
P. Clemens', A. Schumacher ${ }^{2}$ \\ ${ }^{1}$ University of Wuppertal, Faculty 7 \\ Gaußstr. 20, 42119 Wuppertal, Germany \\ pclemens@uni-wuppertal.de \\ ${ }^{2}$ University of Wuppertal, Faculty 7 \\ Gaußstr. 20, 42119 Wuppertal, Germany \\ schumacher@uni-wuppertal.de
}

Key Words: Topology Optimization, Manufacturing Constraint, Sheet Metal, Deep Drawing

\begin{abstract}
Results from topology optimization will only be used in industry if manufacturing considerations have been taken into account. Ideally, the manufacturing processes are already integrated in the optimization process. Dienemann et. al [1] developed a scheme to optimize sheet metal structures by using the deep drawing manufacturing constraints. To do so a surface is calculated that represents the current sheet design and used to penalize the sensitivities of the voxel finite elements depending on their distance to it. This manufacturing constraint can cause the optimization to become stuck in a local optimum far off the global optimum. To prevent this, new methods that change the optimization process have been developed.
\end{abstract}




\section{INTRODUCTION}

In 2015, a method for the topology optimization of sheet metal structures with a manufacturing constraint for deep drawing has been introduced by R. Dienemann, A. Schumacher and A. Fiebig [2]. To ensure a design that can be manufactured by deep drawing in a single step, objective sensitivities are penalized. A surface is used to determine the penalization of the objective sensitivities. The penalization is dependent of the minimum distance of an element to the surface. Penalization of objective sensitivities far away from the surface will be high, while close to it will be low. The penalization is done by multiplying with $P_{i}$. [1]

$$
P_{i}=\frac{1+\exp (-f \cdot a)}{1+\exp \left(2 a / b \cdot\left(d_{i}-f\right)\right)} \quad f=\frac{\ln \left(-2+\exp \left(a \cdot \frac{b}{2}\right)\right)}{a}
$$

The user defines a desired constant wall thickness $b$ and a punch direction. The shortest distance between the element $i$ and the surface is $d_{i}$ and $a$ is the discreteness of the penalty function. The form and location of the surface is defined by the element densities and updated in each iteration. Due to this adaptation the surface can move and change shape during the optimization process. To ensure deep drawing of the design, the surface will never have any undercuts. [1]
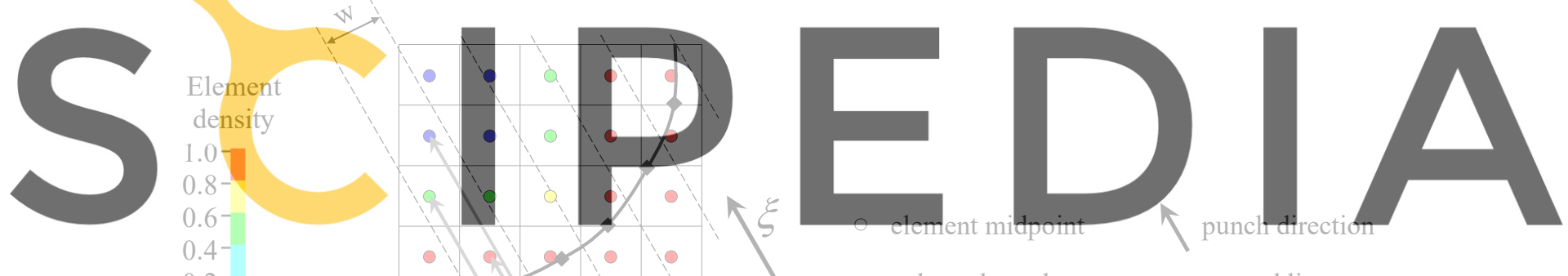

Register for free at https/d WW W. Scipedia.com to download the version without the watermark $\longleftarrow$ element position $\xi_{i} \diamond$ point of mid surface

Figure 1: Calculation of mid surface [1]

Figure 1 shows the calculation of the surface at an exemplary cross section. The surface point per column of width $\mathrm{w}$ is calculated by averaging the element position $\xi_{i}$ with the element densities $x_{i}$ of all elements, which element midpoint is located within the column. [1]

$$
\xi_{m}=\frac{\sum \xi_{i} \cdot x_{i}}{\sum x_{i}}
$$

The penalization of the objective sensitivities leads to a shell structure [1]. Because the elements with a distance half of the desired wall thickness to the surface are not or weakly penalized, a shell structure of the desired wall thickness is very likely to be the optimization result. 


\section{EXAMPLES OF FAILED OPTIMIZATIONS}

Obtaining sheet metal designs with deep draw constraints has been demonstrated [1] and can probably be achieved for most linear topology optimization problems. However, the method is prone to stuck in local optima far off the global optimum because of the deep draw constraint. The following two examples demonstrate optimization results stuck in such poor local optimum. The finite elements used are voxel elements with 8 nodes. The displayed single point constraints (SPC) are blocking all 6 degrees of freedom. The material properties are those of steel with a Young Modulus of $210000 \mathrm{~N} / \mathrm{mm}^{2}$ and a Poisson Ratio of 0.3 . The optimizations are performed by using the method for topology optimization with deep draw constraint presented by Dienemann et. al [1] and OptiStruct from Altair as solver.

\section{Example 1:}
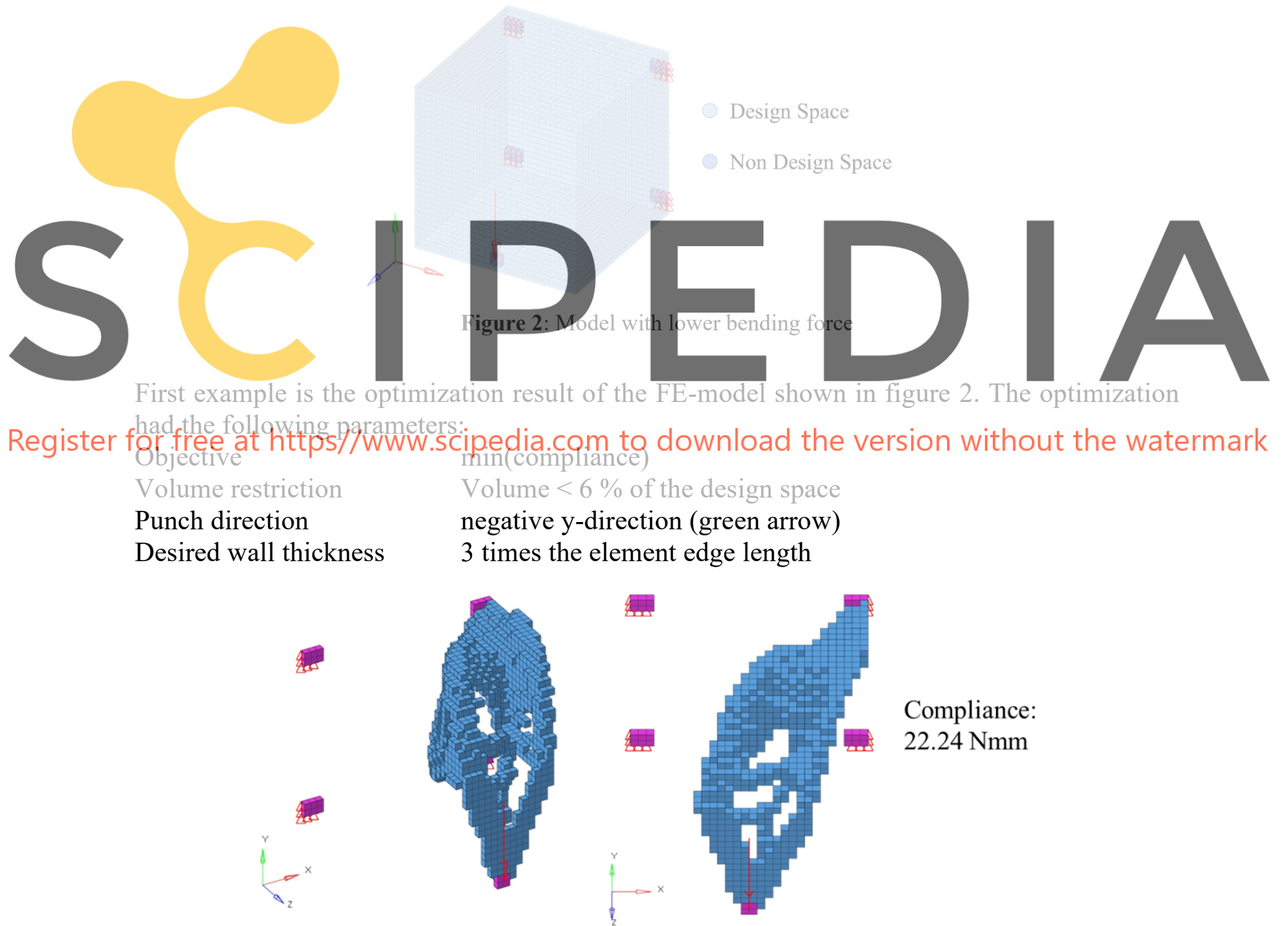

Compliance:

$22.24 \mathrm{Nmm}$

Figure 3: Optimization result (of model in figure 2) 
The result (figure 3) is clearly not optimal. The shape is not symmetrical, although the load case and model are. There is only one connection to a SPC group. As will be shown later, close to 6 percent of the design space volume is enough to allow for a symmetrical design and the performance can be significantly improved by connecting more of the given SPC's to the structure. What makes the optimization with a deep draw constraint difficult for this model is the positions of the SPC's. This can be shown with the optimization of the model given in figure 4 , if the optimization is done with the same configuration as before. As presented in figure 4, this model is only different in terms of SPC's.

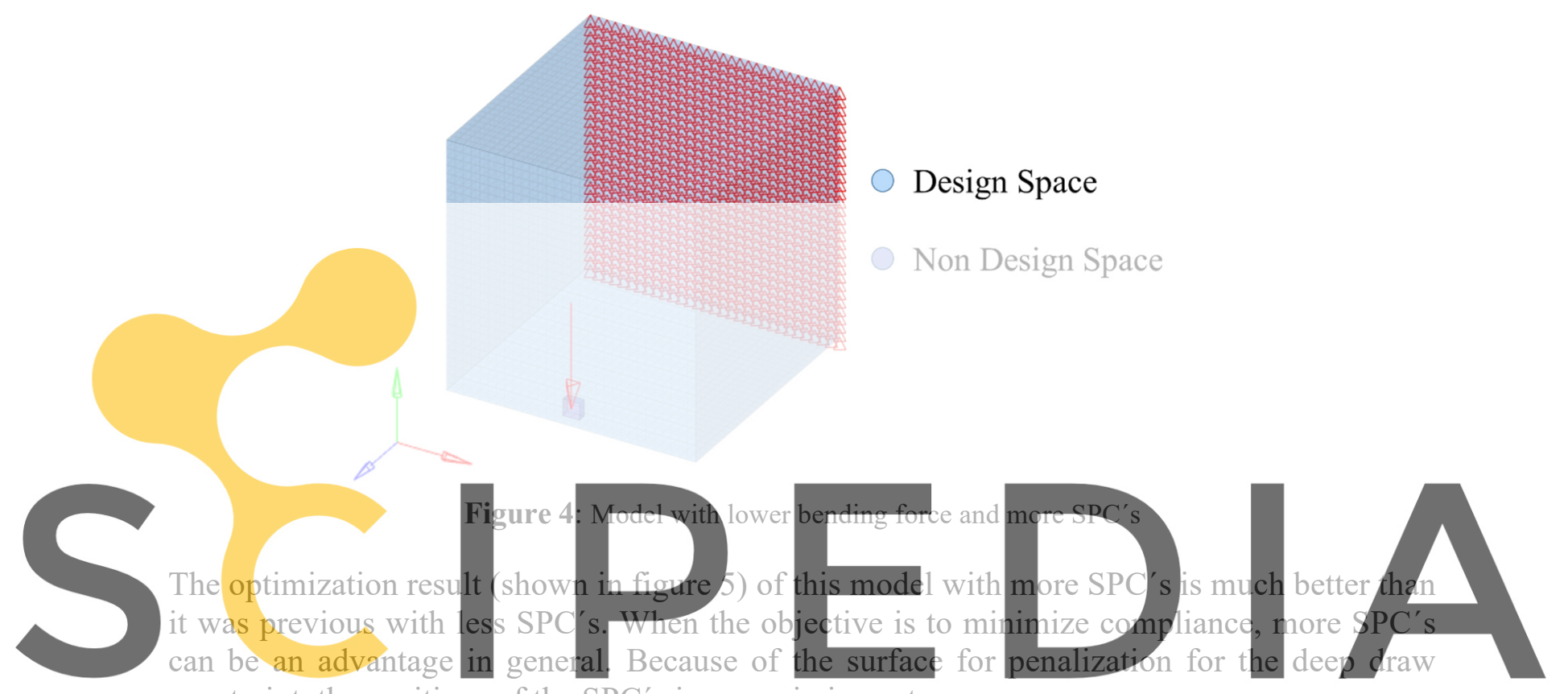

constraint, the positions of the SPC's increase in importance.

Register for free at https//www.scipedia.com to download the version without the watermark

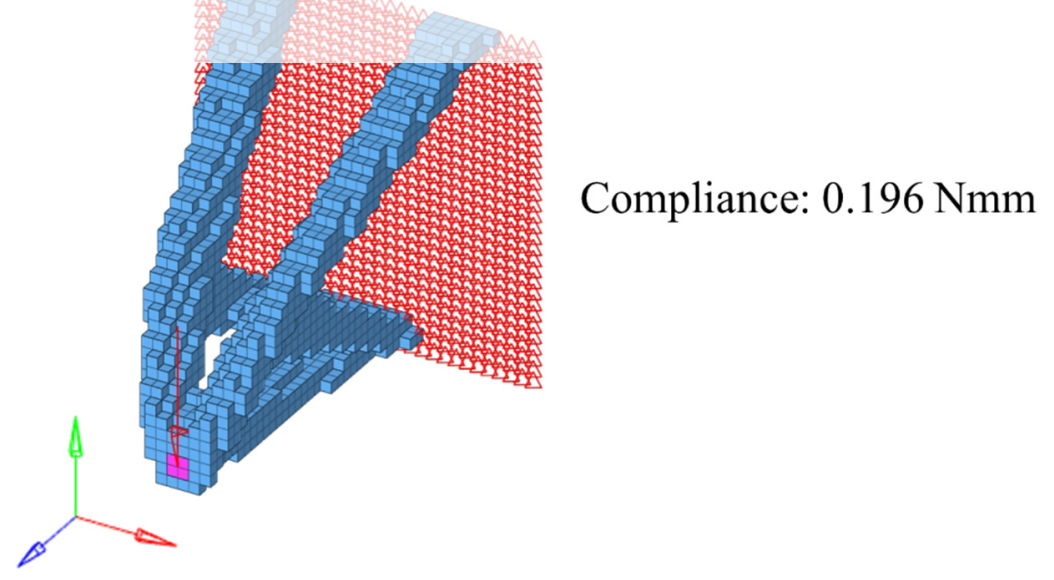

Figure 5: Optimization result (of model in figure 4)

The initial surfaces for penalization for the models with lower bending force are shown in figure 6. With SPC's only in the corners of the model and a punch direction in negative y- 
direction the initial surface doesn't have any SPC in its close environment. The distance between SPC's and surface results in penalization of the objective sensitivities near the SPC's. In contrast, with more SPC's in the second model, the surface is always in touch with SPC's.

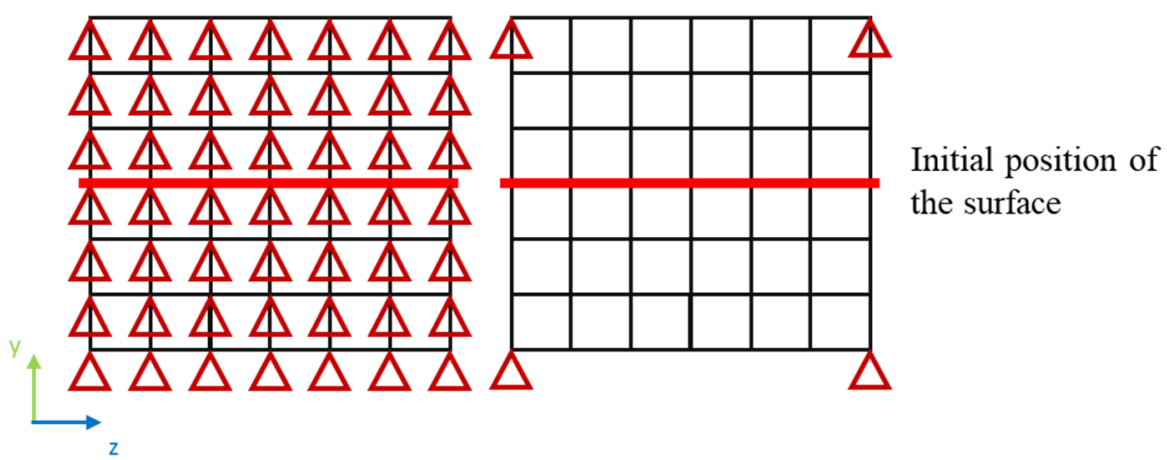

Figure 6: Initial positions of the surface for penalization

\section{Example 2:}
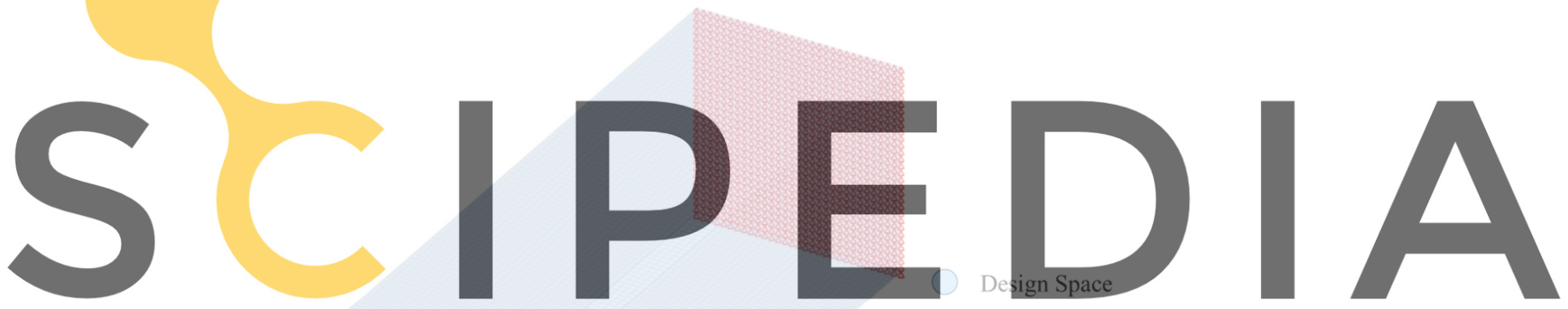

Register for free at https//www.scipedia.com to download the wersi@in without the watermark

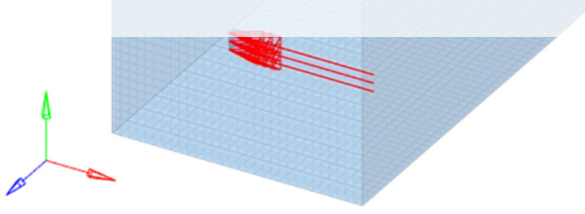

Figure 7: Model with central bending force

Second example is the optimization result of the FE-model shown in figure 7 with the following optimization parameters:

Objective

Volume restriction

Punch direction

Desired wall thickness $\min ($ compliance)

Volume $<22 \%$ of the design space negative y-direction (green arrow) 3 times the element edge length 


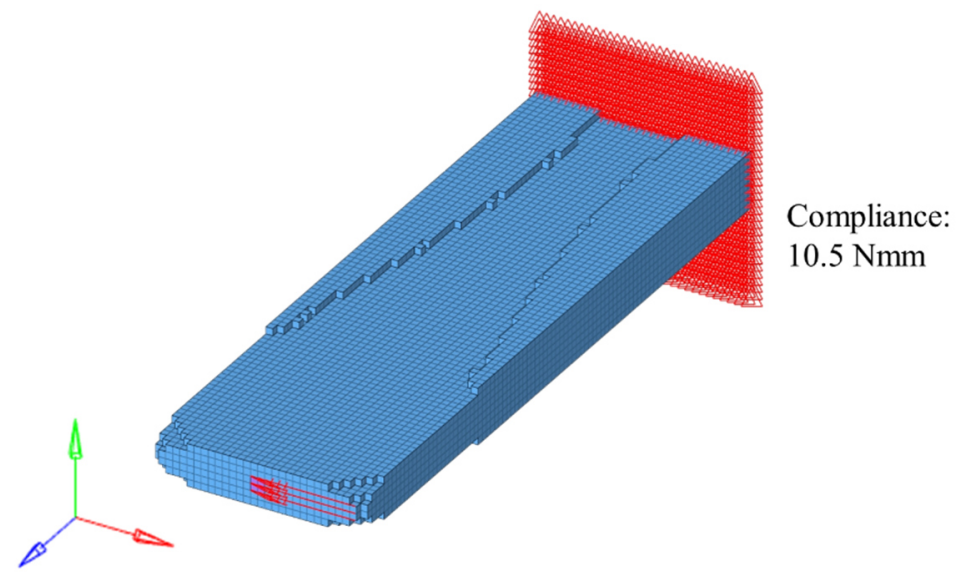

Figure 8: Optimization result

Figure 8 shows the optimization result. For an engineer it is easy to see that this design is not optimal. For this load and geometry, a good design (without the deep draw constraint involved) would be a H-Beam [3]. Because of the deep draw constraint an H-Beam is not part of the feasible solution space. A still much better performing deep drawable design within the feasible design space would be a U-profile. This can be checked by doing two comparable

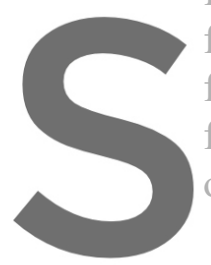
finite element analys es. force. The model on the figure 8 , on the right objective, the U-Profile
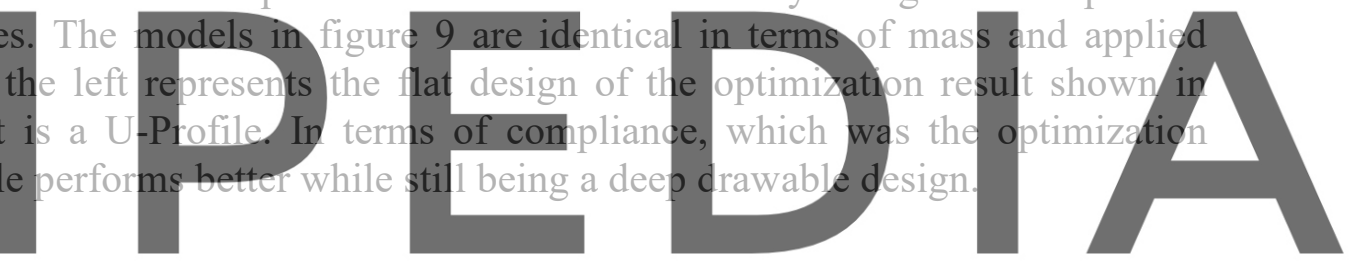

Register for free at https//www.scipedia.com to download the version without the watermark

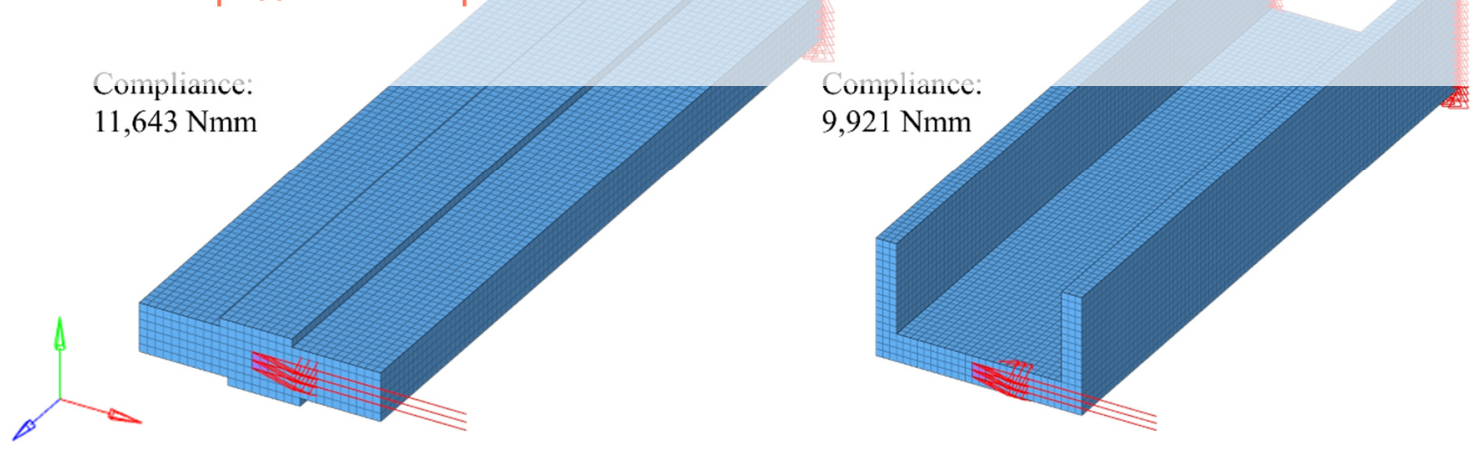

Figure 9: Optimization result

It has become clear that the optimization result should be more like a U-profile than a flat structure. The reason why the optimization result isn't shaped like a U-profile will be discussed in detail in chapter 4 . The main issue is how the surface for penalization changes during optimization. 


\section{ADAPTIV WALL THICKNESS}

By using the deep draw manufacturing constraint, depending on model size and desired wall thickness most of the objective sensitivities are penalized for the whole optimization process from the beginning. Because of this the process is very sensitive to the initial position of the surface used to determine penalization. To counteract this, an adaptive wall thickness can be used. Usually, the engineer will select a desired wall thickness well below the thickness of the design space. Therefore, only a tiny sector of the design space will not be punished by the manufacturing constraint. To prevent this, the presented method changes the sheet thickness aimed for during the optimization which will change the penalty of the objective sensitivities. By starting with a very high wall thickness, in the beginning the optimization is closer to an optimization without the manufacturing constraint. Most of the objective sensitivities doesn't get penalized. The topology optimization for sheet metal parts aims for a specific sheet thickness. To take this into account, over the optimization process the wall thickness is reduced step by step until it reaches the desired wall thickness given by the user for the shell design.

Chances to find an optimum that performs closer to the global optimum is increased. During the less constraint phase of the optimization, the design can change more easily and a lot of points that would be local optima with a thin wall thickness aren't at this point of the optimization. So before finding the closest local optima for a shell structure, the optimization will move towards a more general local optimum first. When the desired wall thickness is

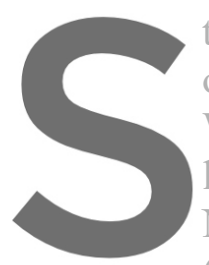
then reduced, a local op optima will be searchec When the adaptive wall thickness is applied lower bending force the Nmm (result shown (right).
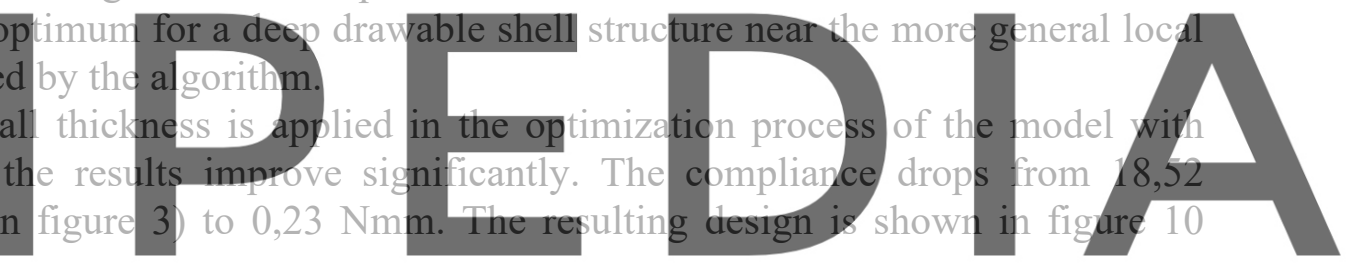

Register for free at https//www.scipedia.com to download the version without the watermark
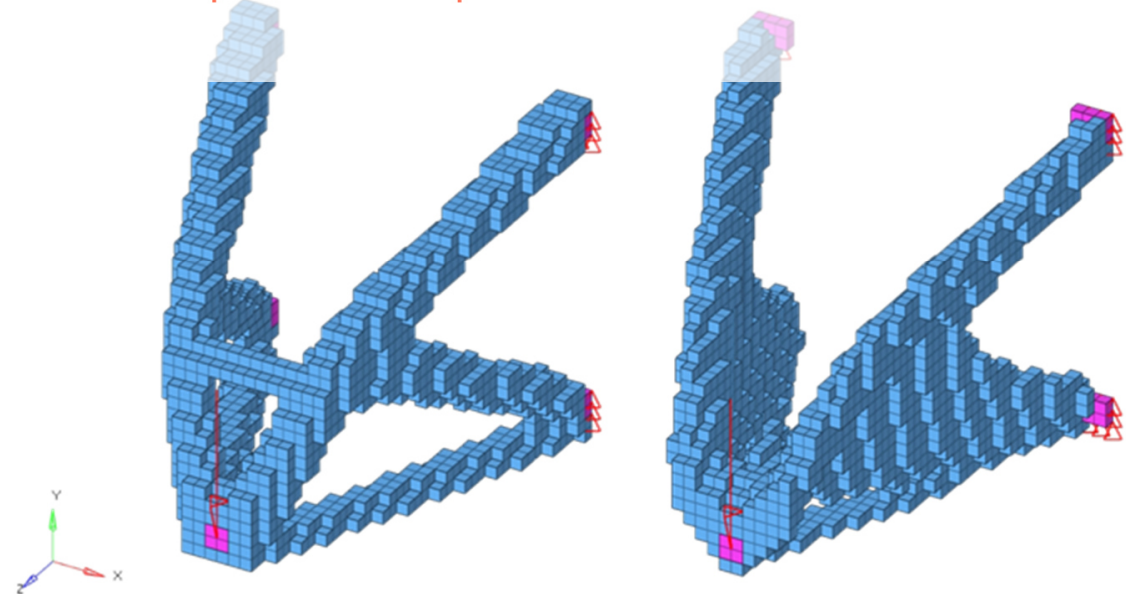

Compliance:

$0.23 \mathrm{Nmm}$

Figure 10: Optimization result without deep draw constraint (left) and with deep draw constraint and adaptive wall thickness (right) 
As shown in figure 10, using the adaptive wall thickness can result in a design very close to the design that results if a topology optimization without deep draw constraint is performed. Independent of the user input, if using the adaptive wall thickness the initial wall thickness is set to $60 \%$ of the design space length in punch direction. When performing topology optimization with deep draw constraint, it is preferable to use the continuation method, which changes the penalty factor during the optimization process [1][4]. While the current wall thickness is not equal to the desired one, the wall thickness will be reduced every time the penalty factor is changed during the optimization. In the beginning of the optimization process the wall thickness is reduced fast, later the reduction is slowed down. For the optimization of the model with lower bending force, the progression of the wall thickness through the optimization process is shown in figure 11 , the desired wall thickness being set to 3 times the element edge length.

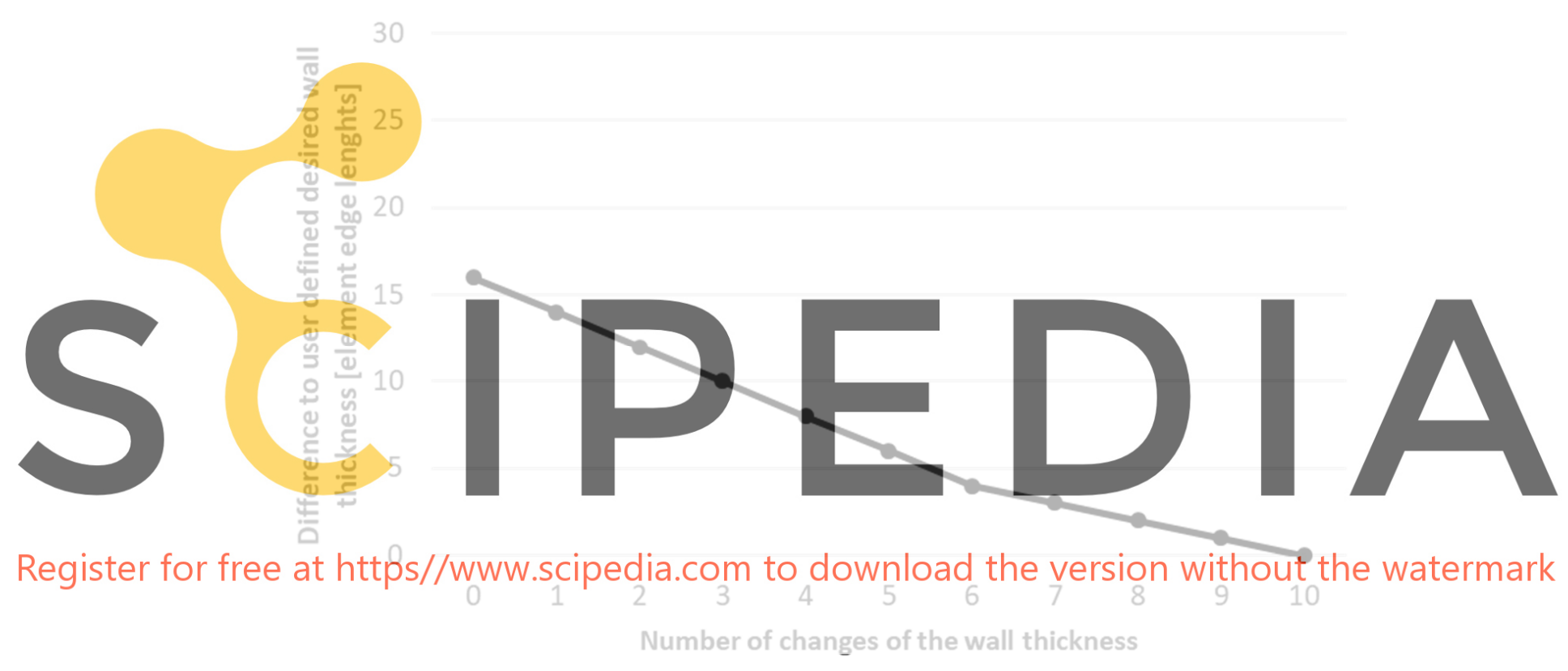

Figure 11: Wall thickness during optimization 


\section{PENALIZATION FREE MODEL EDGES}

The way the surface for penalization is calculated holds a problem. The sheet metal optimization is not able to find designs where larger parts of the sheet metal are orientated in the exact same direction of the punch direction. The surface used for the manufacturing constraint and the way it is calculated is the reason for this. As soon as there are larger parts of material oriented in the deep draw direction, the constraint surface will begin to change shape in these regions. The objective sensitivities will be punished in such a way that the parts of the structure orientated in punch direction will vanish in the following iterations. The process of these structures disappearing during the optimization is illustrated below in figure 12 .

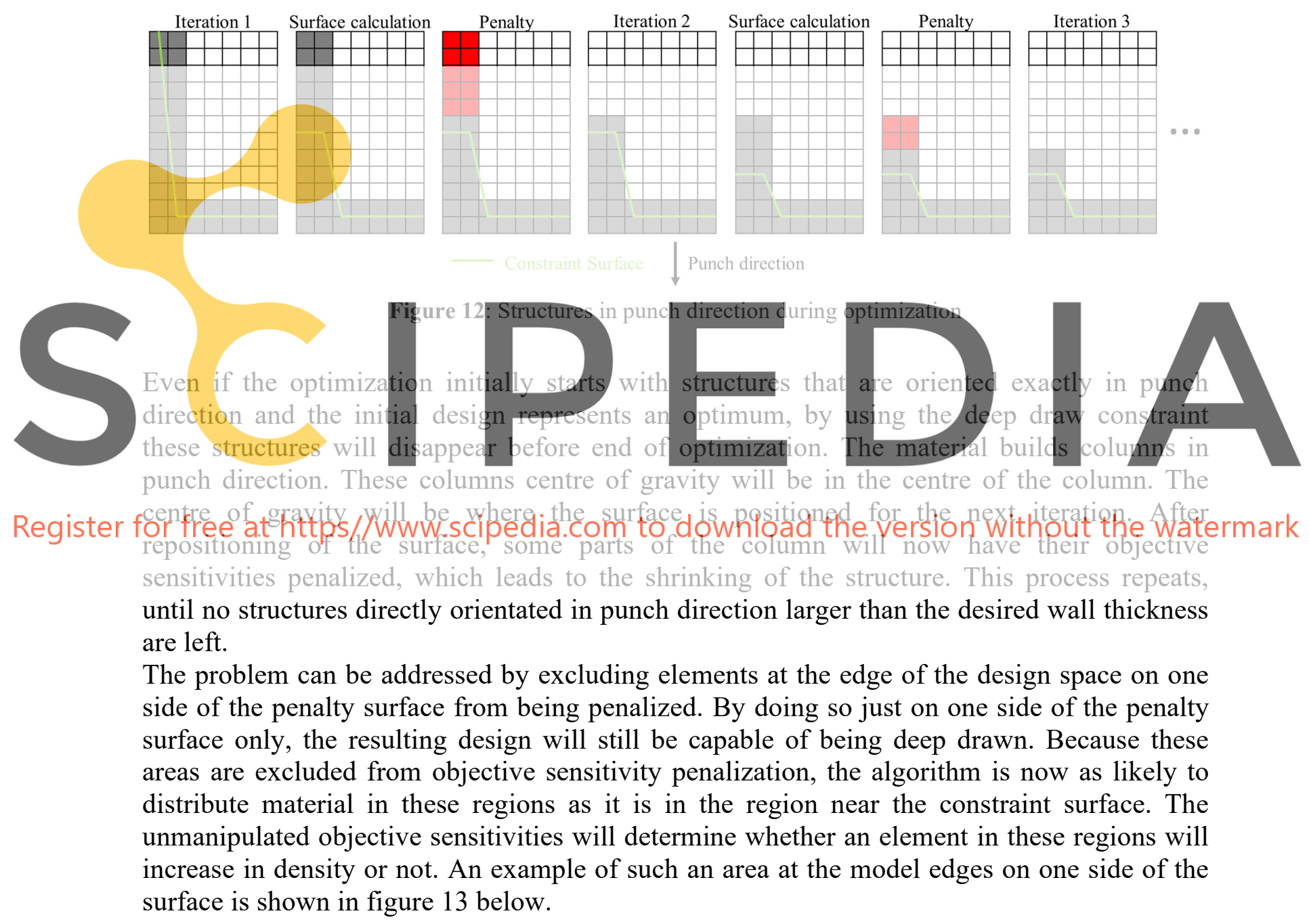




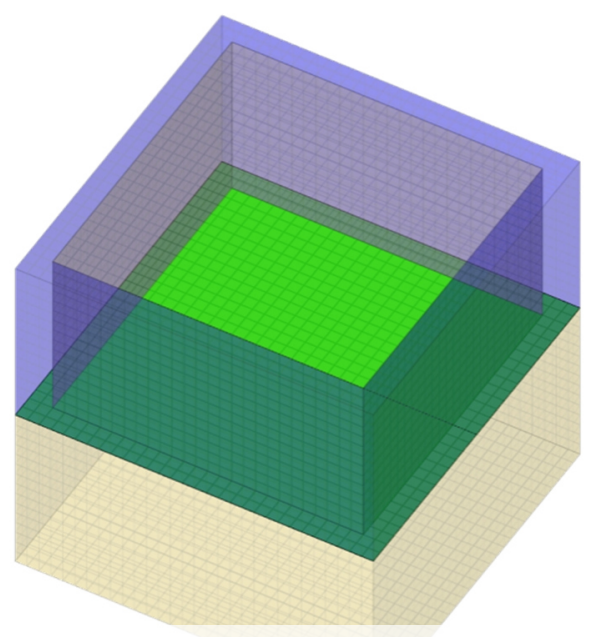

Figure 13: Example of penalization free edges (blue), surface (green)

Which elements are excluded from objective sensitivities penalization must be evaluated every iteration during optimization because of the moving surface for penalization. Applied to the optimization of the model with central bending force, the penalization free model edges

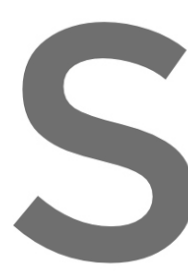
lead to the expected compliance is reduced
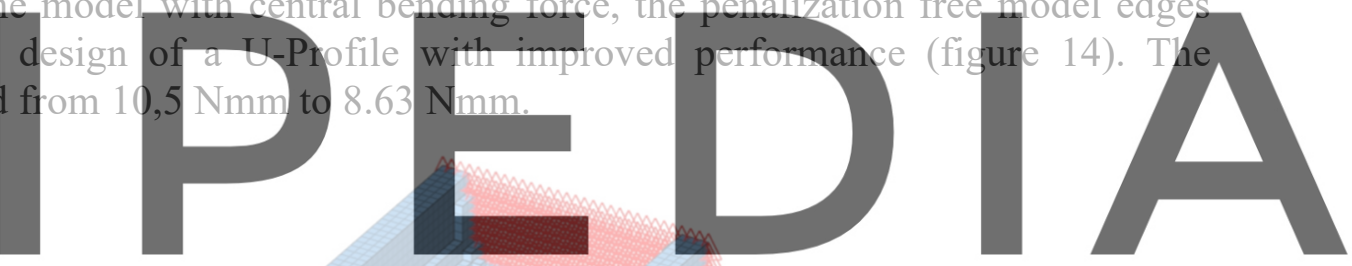

Register for free at https//www.scipedia.com to download the version without the watermark

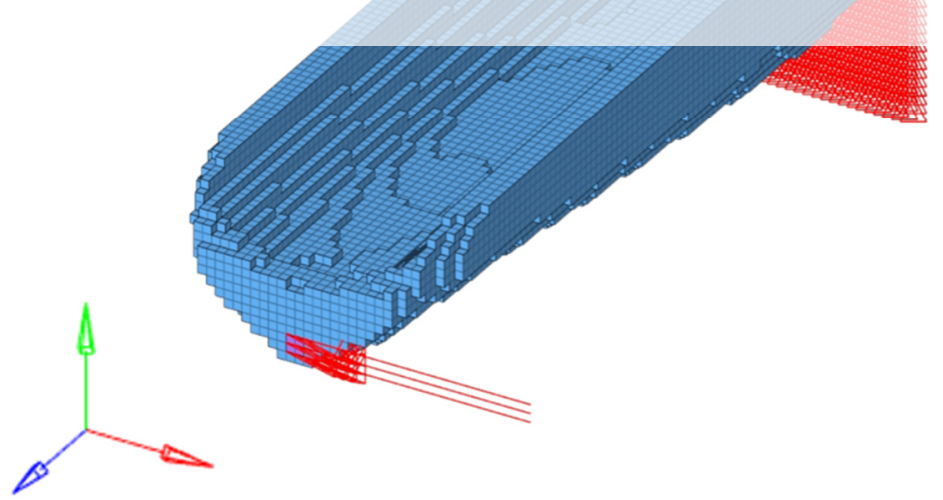

Compliance:

$8.63 \mathrm{Nmm}$

Figure 14: Model with central bending force (figure 7) optimization result with penalization free model edges

Of course, even with this method, it is still not possible to obtain designs with structures in punch direction in the center of the design space. To improve geometrical moment of inertia in case of a bending force the edges are the area of interest. 


\section{CONCLUSIONS}

In some cases, the optimization with the deep draw constraint [1] can lead the optimization to get stuck in poor local optima. For some of these cases, the presented methods can improve the optimization results significantly.

With the adaptive wall thickness the optimization behave more like topology optimization without deep draw constraint in the beginning of the optimization. It then becomes more restrictive with each iteration. For optimization problems ending up in a poor local optimum, this method can lead to a better design.

The second presented method of excluding the model edges from penalization can be especially effective for bending force load cases. By excluding the edges of the design space on one side of the deep draw constraint surface, it becomes possible to find designs with structures that are orientated in punch direction and still able to be deep drawn.

\section{REFERENCES}

[1] Dienemann, R.; Schumacher, A.; Fiebig, S. 2017: Topology optimization for finding shell structures manufactured by deep drawing, Struct Multidisc Optim (2017) 56:473-485

[2] Dienemann, R.; Schumacher, A.; Fiebig, S. 2015: Topology optimization considering the requirements of deep-drawn sheet metals, Proc. of 11 th World Congress on Structural and Multidisciplinary Optimization, Sydney, Australia

[3] Beitz, Wolfgang / Küttner, Karl-Heinz (2013): DUBBEL - Handbook of Mechanical Engineering. Berlin Heidelberg (Springer Science \& Business Media)

[4] S. Rojas-Labanda and M. Stolpe, Automatic penalty continuation in structural topology optimization, Structural and Multidisciplinary Optimization, 52, 1205-1221, 2015 